\title{
Fractional Calculus of Variations in Terms of a Generalized Fractional Integral with Applications to Physics
}

\author{
Tatiana Odzijewicz, ${ }^{1}$ Agnieszka B. Malinowska, ${ }^{2}$ \\ and Delfim F. M. Torres ${ }^{1}$ \\ ${ }^{1}$ Center for Research and Development in Mathematics and Applications, Department of Mathematics, \\ University of Aveiro, 3810-193 Aveiro, Portugal \\ ${ }^{2}$ Faculty of Computer Science, Białystok University of Technology, 15-351 Białystok, Poland
}

Correspondence should be addressed to Delfim F. M. Torres, delfim@ua.pt

Received 1 January 2012; Revised 25 February 2012; Accepted 27 February 2012

Academic Editor: Muhammad Aslam Noor

Copyright (C 2012 Tatiana Odzijewicz et al. This is an open access article distributed under the Creative Commons Attribution License, which permits unrestricted use, distribution, and reproduction in any medium, provided the original work is properly cited.

We study fractional variational problems in terms of a generalized fractional integral with Lagrangians depending on classical derivatives, generalized fractional integrals and derivatives. We obtain necessary optimality conditions for the basic and isoperimetric problems, as well as natural boundary conditions for free-boundary value problems. The fractional action-like variational approach (FALVA) is extended and some applications to physics discussed.

\section{Introduction}

The calculus of variations is a beautiful and useful field of mathematics that deals with problems of determining extrema (maxima or minima) of functionals [1-3]. It starts with the simplest problem of finding a function extremizing (minimizing or maximizing) an integral

$$
\partial(y)=\int_{a}^{b} F\left(t, y(t), y^{\prime}(t)\right) d t
$$

subject to boundary conditions $y(a)=y_{a}$ and $y(b)=y_{b}$. In the literature, many generalizations of this problem were proposed, including problems with multiple integrals, functionals containing higher-order derivatives, and functionals depending on several 
functions [4-6]. Of our interest is an extension proposed by Riewe in 1996-1997, where fractional derivatives (real or complex order) are introduced in the Lagrangian $[7,8]$.

During the last decade, fractional problems have increasingly attracted the attention of many researchers. As mentioned in [9], Science Watch of Thomson Reuters identified the subject as an Emerging Research Front area. Fractional derivatives are nonlocal operators and are historically applied in the study of nonlocal or time-dependent processes [10]. The first and well-established application of fractional calculus in physics was in the framework of anomalous diffusion, which is related to features observed in many physical systems. Here we can mention the report [11] demonstrating that fractional equations work as a complementary tool in the description of anomalous transport processes. Within the fractional approach, it is possible to include external fields in a straightforward manner. As a consequence, in a short period of time, the list of applications expanded. Applications include chaotic dynamics [12], material sciences [13], mechanics of fractal and complex media [14, 15], quantum mechanics [16, 17], physical kinetics [18], long-range dissipation [19], and long-range interaction [20,21], just to mention a few. One of the most remarkable applications of fractional calculus appears, however, in the fractional variational calculus, in the context of classical mechanics. Riewe $[7,8]$ shows that a Lagrangian involving fractional time derivatives leads to an equation of motion with nonconservative forces such as friction. It is a remarkable result since frictional and nonconservative forces are beyond the usual macroscopic variational treatment and, consequently, beyond the most advanced methods of classical mechanics [22]. Riewe generalizes the usual variational calculus, by considering Lagrangians that are dependent on fractional derivatives, in order to deal with nonconservative forces. Recently, several different approaches have been developed to generalize the least action principle and the Euler-Lagrange equations to include fractional derivatives. Results include problems depending on Caputo fractional derivatives and Riemann-Liouville fractional derivatives [23-35].

A more general unifying perspective to the subject is, however, possible, by considering fractional operators depending on general kernels [36-38]. In this work, we follow such an approach, developing a generalized fractional calculus of variations. We consider very general problems, where the classical integrals are substituted by generalized fractional integrals, and the Lagrangians depend not only on classical derivatives but also on generalized fractional operators. Problems of the type considered here, for particular kernels, are important in physics [39]. Here, we obtain general necessary optimality conditions, for several types of variational problems, which are valid for rather arbitrary operators and kernels. By choosing particular operators and kernels, one obtains the recent results available in the literature of mathematical physics [39-44].

The paper is organized as follows. In Section 2, we introduce the generalized fractional operators and prove some of their basic properties. Section 3 is dedicated to prove integration by parts formulas for the generalized fractional operators. Such formulas are then used in later sections to prove necessary optimality conditions (Theorems 4.2 and 6.3). In Sections 4,5 , and 6 we study three important classes of generalized variational problems: we obtain fractional Euler-Lagrange conditions for the fundamental (Section 4) and generalized isoperimetric problems (Section 6), as well as fractional natural boundary conditions for generalized free-boundary value problems (Section 5). Finally, two illustrative examples are discussed in detail in Section 7, while applications to physics are given in Section 8: in Section 8.1, we obtain the damped harmonic oscillator in quantum mechanics; in Section 8.2, we show how results from FALVA physics can be obtained. We end with Section 9 of conclusion, pointing out an important direction of future research. 


\section{Preliminaries}

In this section, we present definitions and properties of generalized fractional operators. As particular cases, by choosing appropriate kernels, these operators are reduced to standard fractional integrals and fractional derivatives. Other nonstandard kernels can also be considered as particular cases. For more on the subject of generalized fractional calculus and applications, we refer the reader to [37]. Throughout the text, $\alpha$ denotes a real number between zero and one. Following [45], we use round brackets for the arguments of functions and square brackets for the arguments of operators. By definition, an operator receives and returns a function.

Definition 2.1 (generalized fractional integral). The operator $K_{P}^{\alpha}$ is given by

$$
K_{P}^{\alpha}[f](x)=K_{P}^{\alpha}[t \longmapsto f(t)](x)=p \int_{a}^{x} k_{\alpha}(x, t) f(t) d t+q \int_{x}^{b} k_{\alpha}(t, x) f(t) d t
$$

where $P=\langle a, x, b, p, q\rangle$ is the parameter set ( $p$-set for brevity), $x \in[a, b], p, q$ are real numbers, and $k_{\alpha}(x, t)$ is a kernel which may depend on $\alpha$. The operator $K_{P}^{\alpha}$ is referred to as the operator $K$ (K-op for simplicity) of order $\alpha$ and $p$-set $P$, while $K_{P}^{\alpha}[f]$ is called the operation $K$ (or $K$-opn) of $f$ of order $\alpha$ and $p$-set $P$.

Note that if we define

$$
G(x, t):= \begin{cases}p k_{\alpha}(x, t), & \text { if } t<x, \\ q k_{\alpha}(t, x), & \text { if } t \geq x,\end{cases}
$$

then the operator $K_{P}^{\alpha}$ can be written in the form

$$
K_{P}^{\alpha}[f](x)=K_{P}^{\alpha}[t \longmapsto f(t)](x)=\int_{a}^{b} G(x, t) f(t) d t .
$$

This is a particular case of one of the oldest and most respectable class of operators, the socalled Fredholm operators [46, 47].

Theorem 2.2 (cf. Example 6 of [46]). Let $\alpha \in(0,1)$ and $P=\langle a, x, b, p, q\rangle$. If $k_{\alpha}$ is a square integrable function on the square $\Delta=[a, b] \times[a, b]$, then $K_{P}^{\alpha}: L_{2}([a, b]) \rightarrow L_{2}([a, b])$ is welldefined, linear, and bounded operator.

Theorem 2.3. Let $k_{\alpha}$ be a difference kernel, that is, let $k_{\alpha} \in L_{1}([a, b])$ with $k_{\alpha}(x, t)=k_{\alpha}(x-t)$. Then, $K_{P}^{\alpha}: L_{1}([a, b]) \rightarrow L_{1}([a, b])$ is a well defined bounded and linear operator.

Proof. Obviously, the operator is linear. Let $\alpha \in(0,1), P=\langle a, t, b, p, q\rangle$, and $f \in L_{1}([a, b])$. Define

$$
F(\tau, t):= \begin{cases}\left|p k_{\alpha}(t-\tau)\right| \cdot|f(\tau)|, & \text { if } \tau \leq t \\ \left|q k_{\alpha}(\tau-t)\right| \cdot|f(\tau)|, & \text { if } \tau>t\end{cases}
$$


for all $(\tau, t) \in \Delta=[a, b] \times[a, b]$. Since $F$ is measurable on the square $\Delta$, we have

$$
\begin{aligned}
\int_{a}^{b}\left(\int_{a}^{b} F(\tau, t) d t\right) d \tau & =\int_{a}^{b}\left[|f(\tau)|\left(\int_{\tau}^{b}\left|p k_{\alpha}(t-\tau)\right| d t+\int_{a}^{\tau}\left|q k_{\alpha}(\tau-t)\right| d t\right)\right] d \tau \\
& \leq \int_{a}^{b}|f(\tau)|\|p|-| q||\| k_{\alpha} \| d \tau \\
& =\|p|-| q|| \cdot\| k_{\alpha}\|\cdot\| f \| .
\end{aligned}
$$

It follows from Fubini's theorem that $F$ is integrable on the square $\Delta$. Moreover,

$$
\begin{aligned}
\left\|K_{P}^{\alpha}[f]\right\| & =\int_{a}^{b}\left|p \int_{a}^{t} k_{\alpha}(t-\tau) f(\tau) d \tau+q \int_{t}^{b} k_{\alpha}(\tau-t) f(\tau) d \tau\right| d t \\
& \leq \int_{a}^{b}\left(|p| \int_{a}^{t}\left|k_{\alpha}(t-\tau)\right| \cdot|f(\tau)| d \tau+|q| \int_{t}^{b}\left|k_{\alpha}(\tau-t)\right| \cdot|f(\tau)| d \tau\right) d t \\
& =\int_{a}^{b}\left(\int_{a}^{b} F(\tau, t) d \tau\right) d t \\
& \leq\|p|-| q|| \cdot\| k_{\alpha}\|\cdot\| f \| .
\end{aligned}
$$

Hence, $K_{P}^{\alpha}: L_{1}([a, b]) \rightarrow L_{1}([a, b])$ and $\left\|K_{P}^{\alpha}\right\| \leq\|p|-| q\| \cdot\left\|k_{\alpha}\right\|$.

Remark 2.4. The $K$-op reduces to the left and the right Riemann-Liouville fractional integrals from a suitably chosen kernel $k_{\alpha}(x, t)$ and $p$-set $P$. Let $k_{\alpha}(x, t)=k_{\alpha}(x-t)=(1 / \Gamma(\alpha))(x-t)^{\alpha-1}$ :

(i) if $P=\langle a, x, b, 1,0\rangle$, then

$$
K_{P}^{\alpha}[f](x)=\frac{1}{\Gamma(\alpha)} \int_{a}^{x}(x-t)^{\alpha-1} f(t) d t=:{ }_{a} I_{x}^{\alpha}[f](x)
$$

is the standard left Riemann-Liouville fractional integral of $f$ of order $\alpha$;

(ii) if $P=\langle a, x, b, 0,1\rangle$, then

$$
K_{P}^{\alpha}[f](x)=\frac{1}{\Gamma(\alpha)} \int_{x}^{b}(t-x)^{\alpha-1} f(t) d t=:{ }_{x} I_{b}^{\alpha}[f](x)
$$

is the standard right Riemann-Liouville fractional integral of $f$ of order $\alpha$.

Corollary 2.5. Operators ${ }_{a} I_{x}^{\alpha},{ }_{x} I_{b}^{\alpha}: L_{1}([a, b]) \rightarrow L_{1}([a, b])$ are well defined, linear and bounded.

The generalized fractional derivatives $A_{P}^{\alpha}$ and $B_{P}^{\alpha}$ are defined in terms of the generalized fractional integral $K$-op. 
Definition 2.6 (generalized Riemann-Liouville fractional derivative). Let $P$ be a given parameter set and $0<\alpha<1$. The operator $A_{P}^{\alpha}$ is defined by $A_{P}^{\alpha}=D \circ K_{P}^{1-\alpha}$, where $D$ denotes the standard derivative operator, and is referred to as the operator $A$ ( $A$-op) of order $\alpha$ and $p$-set $P$, while $A_{P}^{\alpha}[f]$, for a function $f$ such that $K_{P}^{1-\alpha}[f] \in A C([a, b])$, is called the operation $A$ (A-opn) of $f$ of order $\alpha$ and $p$-set $P$.

Definition 2.7 (generalized Caputo fractional derivative). Let $P$ be a given parameter set and $\alpha \in(0,1)$. The operator $B_{P}^{\alpha}$ is defined by $B_{P}^{\alpha}=K_{P}^{1-\alpha} \circ D$, where $D$ denotes the standard derivative operator, and is referred to as the operator $B$ (B-op) of order $\alpha$ and $p$-set $P$, while $B_{P}^{\alpha}[f]$, for a function $f \in A C([a, b])$, is called the operation $B$ (B-opn) of $f$ of order $\alpha$ and $p$-set $P$.

Remark 2.8. The standard Riemann-Liouville and Caputo fractional derivatives are easily obtained from the generalized operators $A_{P}^{\alpha}$ and $B_{P}^{\alpha}$, respectively. Let $k_{1-\alpha}(x, t)=k_{1-\alpha}(x-t)=$ $(x-t)^{-\alpha} / \Gamma(1-\alpha)$ :

(i) if $P=\langle a, x, b, 1,0\rangle$, then

$$
A_{P}^{\alpha}[f](x)=\frac{1}{\Gamma(1-\alpha)} D\left[\xi \longmapsto \int_{a}^{\xi}(\xi-t)^{-\alpha} f(t) d t\right](x)=:_{a} D_{x}^{\alpha}[f](x)
$$

is the standard left Riemann-Liouville fractional derivative of $f$ of order $\alpha$, while

$$
B_{P}^{\alpha}[f](x)=\frac{1}{\Gamma(1-\alpha)} \int_{a}^{x}(x-t)^{-\alpha} D[f](t) d t=:_{a}^{C} D_{x}^{\alpha}[f](x)
$$

is the standard left Caputo fractional derivative of $f$ of order $\alpha$;

(ii) if $P=\langle a, x, b, 0,1\rangle$, then

$$
-A_{P}^{\alpha}[f](x)=\frac{-1}{\Gamma(1-\alpha)} D\left[\xi \longmapsto \int_{\xi}^{b}(t-\xi)^{-\alpha} f(t) d t\right](x)={ }_{x} D_{b}^{\alpha}[f](x)
$$

is the standard right Riemann-Liouville fractional derivative of $f$ of order $\alpha$, while

$$
-B_{P}^{\alpha}[f](x)=\frac{-1}{\Gamma(1-\alpha)} \int_{x}^{b}(t-x)^{-\alpha} D[f](t) d t={ }_{:}^{C} D_{b}^{\alpha}[f](x)
$$

is the standard right Caputo fractional derivative of $f$ of order $\alpha$.

\section{On Generalized Fractional Integration by Parts}

We now prove integration by parts formulas for generalized fractional operators. 
Theorem 3.1 (fractional integration by parts for the $K$-op). Let $\alpha \in(0,1), P=\langle a, t, b, p, q\rangle, k_{\alpha}$ be a square-integrable function on $\Delta=[a, b] \times[a, b]$, and $f, g \in L_{2}([a, b])$. The generalized fractional integral $K_{P}^{\alpha}$ satisfies the integration by parts formula

$$
\int_{a}^{b} g(x) K_{P}^{\alpha}[f](x) d x=\int_{a}^{b} f(x) K_{P^{*}}^{\alpha}[g](x) d x
$$

where $P^{*}=\langle a, t, b, q, p\rangle$.

Proof. Define

$$
F(\tau, t):= \begin{cases}\left|p k_{\alpha}(t, \tau)\right| \cdot|g(t)| \cdot|f(\tau)|, & \text { if } \tau \leq t, \\ \left|q k_{\alpha}(\tau, t)\right| \cdot|g(t)| \cdot|f(\tau)|, & \text { if } \tau>t,\end{cases}
$$

for all $(\tau, t) \in \Delta$. Applying Holder's inequality, we obtain

$$
\begin{aligned}
& \int_{a}^{b}\left(\int_{a}^{b} F(\tau, t) d t\right) d \tau=\int_{a}^{b}\left[|f(\tau)|\left(\int_{\tau}^{b}\left|p k_{\alpha}(t, \tau)\right| \cdot|g(t)| d t+\int_{a}^{\tau}\left|q k_{\alpha}(\tau, t)\right| \cdot|g(t)| d t\right)\right] d \tau \\
& \leq \int_{a}^{b}\left[|f(\tau)|\left(\int_{a}^{b}\left|p k_{\alpha}(t, \tau)\right| \cdot|g(t)| d t+\int_{a}^{b}\left|q k_{\alpha}(\tau, t)\right| \cdot|g(t)| d t\right)\right] d \tau \\
& \leq \int_{a}^{b}\left\{| f ( \tau ) | \left[\left(\int_{a}^{b}\left|p k_{\alpha}(t, \tau)\right|^{2} d t\right)^{1 / 2}\left(\int_{a}^{b}|g(t)|^{2} d t\right)^{1 / 2}\right.\right. \\
&\left.\left.+\left(\int_{a}^{b}\left|q k_{\alpha}(\tau, t)\right|^{2} d t\right)^{1 / 2}\left(\int_{a}^{b}|g(t)|^{2} d t\right)^{1 / 2}\right]\right\} d \tau .
\end{aligned}
$$

By Fubini's theorem, functions $k_{\alpha, \tau}(t):=k_{\alpha}(t, \tau)$ and $\widehat{k}_{\alpha, \tau}(t):=k_{\alpha}(\tau, t)$ belong to $L_{2}([a, b])$ for almost all $\tau \in[a, b]$. Therefore,

$$
\begin{gathered}
\int_{a}^{b}\left\{| f ( \tau ) | \left[\left(\int_{a}^{b}\left|p k_{\alpha}(t, \tau)\right|^{2} d t\right)^{1 / 2}\left(\int_{a}^{b}|g(t)|^{2} d t\right)^{1 / 2}\right.\right. \\
\left.\left.+\left(\int_{a}^{b}\left|q k_{\alpha}(\tau, t)\right|^{2} d t\right)^{1 / 2}\left(\int_{a}^{b}|g(t)|^{2} d t\right)^{1 / 2}\right]\right\} d \tau \\
=\|g\|_{2} \int_{a}^{b}\left[|f(\tau)|\left(\left\|p k_{\alpha, \tau}\right\|_{2}+\left\|q \widehat{k}_{\alpha, \tau}\right\|_{2}\right)\right] d \tau
\end{gathered}
$$


Abstract and Applied Analysis

$$
\begin{aligned}
& \leq\|g\|_{2}\left(\int_{a}^{b}|f(\tau)|^{2} d \tau\right)^{1 / 2}\left(\int_{a}^{b}\left|\left\|p k_{\alpha, \tau}\right\|_{2}+\left\|q \widehat{k}_{\alpha, \tau}\right\|_{2}\right|^{2} d \tau\right)^{1 / 2} \\
& \leq\|g\|_{2} \cdot\|f\|_{2}\left(\left\|p k_{\alpha}\right\|_{2}+\left\|q k_{\alpha}\right\|_{2}\right)<\infty .
\end{aligned}
$$

Hence, we can use again Fubini's theorem to change the order of integration:

$$
\begin{aligned}
\int_{a}^{b} g(t) K_{P}^{\alpha}[f](t) d t & =p \int_{a}^{b} g(t) d t \int_{a}^{t} f(\tau) k_{\alpha}(t, \tau) d \tau+q \int_{a}^{b} g(t) d t \int_{t}^{b} f(\tau) k_{\alpha}(\tau, t) d \tau \\
& =p \int_{a}^{b} f(\tau) d \tau \int_{\tau}^{b} g(t) k_{\alpha}(t, \tau) d t+q \int_{a}^{b} f(\tau) d \tau \int_{a}^{\tau} g(t) k_{\alpha}(\tau, t) d t \\
& =\int_{a}^{b} f(\tau) K_{P^{*}}^{\alpha}[g](\tau) d \tau .
\end{aligned}
$$

Theorem 3.2. Let $0<\alpha<1$ and $P=\langle a, x, b, p, q\rangle$. If $k_{\alpha}(x, t)=k_{\alpha}(x-t), k_{\alpha}, f \in L_{1}([a, b])$, and $g \in C([a, b])$, then the operator $K_{P}^{\alpha}$ satisfies the integration by parts formula (3.1).

Proof. Define

$$
F(t, x):= \begin{cases}\left|p k_{\alpha}(x-t)\right| \cdot|g(x)| \cdot|f(t)|, & \text { if } t \leq x, \\ \left|q k_{\alpha}(t-x)\right| \cdot|g(x)| \cdot|f(t)|, & \text { if } t>x,\end{cases}
$$

for all $(t, x) \in \Delta=[a, b] \times[a, b]$. Since $g$ is a continuous function on $[a, b]$, it is bounded on $[a, b]$, that is, there exists a real number $C>0$ such that $|g(x)| \leq C$ for all $x \in[a, b]$. Therefore,

$$
\begin{aligned}
\int_{a}^{b}\left(\int_{a}^{b} F(t, x) d t\right) d x & =\int_{a}^{b}\left[|f(t)|\left(\int_{t}^{b}\left|p k_{\alpha}(x-t)\right| \cdot|g(x)| d x+\int_{a}^{t}\left|q k_{\alpha}(t-x)\right| \cdot|g(x)| d x\right)\right] d t \\
& \leq \int_{a}^{b}\left[|f(t)|\left(\int_{a}^{b}\left|p k_{\alpha}(x-t)\right| \cdot|g(x)| d x+\int_{a}^{b}\left|q k_{\alpha}(t-x)\right| \cdot|g(x)| d x\right)\right] d t \\
& \leq C \int_{a}^{b}\left[|f(t)|\left(\int_{a}^{b}\left|p k_{\alpha}(x-t)\right| d x+\int_{a}^{b}\left|q k_{\alpha}(t-x)\right| d x\right)\right] d t \\
& =C|| p|-| q||\left\|k_{\alpha}\right\|\|f\|<\infty .
\end{aligned}
$$

Hence, we can use Fubini's theorem to change the order of integration in iterated integrals. 
Theorem 3.3 (generalized fractional integration by parts). Let $\alpha \in(0,1)$ and $P=\langle a, t, b, p, q\rangle$. If functions $f, K_{P^{*}}^{1-\alpha}[g] \in A C([a, b])$, and we are in conditions to use formula (3.1) (Theorem 3.1 or Theorem 3.2), then

$$
\int_{a}^{b} g(x) B_{P}^{\alpha}[f](x) d x=\left.f(x) K_{P^{*}}^{1-\alpha}[g](x)\right|_{a} ^{b}-\int_{a}^{b} f(x) A_{P^{*}}^{\alpha}[g](x) d x
$$

where $P^{*}=\langle a, t, b, q, p\rangle$.

Proof. From Definition 2.7, we know that $B_{P}^{\alpha}[f](x)=K_{P}^{1-\alpha}[D[f]](x)$. It follows that

$$
\int_{a}^{b} g(x) B_{P}^{\alpha}[f](x) d x=\int_{a}^{b} g(x) K_{P}^{1-\alpha}[D[f]](x) d x
$$

By relation (3.1),

$$
\int_{a}^{b} g(x) B_{P}^{\alpha}[f](x) d x=\int_{a}^{b} D[f](x) K_{P^{*}}^{1-\alpha}[g](x) d x
$$

and the standard integration by parts formula implies (3.8):

$$
\int_{a}^{b} g(x) B_{P}^{\alpha}[f](x) d x=\left.f(x) K_{P^{*}}^{1-\alpha}[g](x)\right|_{a} ^{b}-\int_{a}^{b} f(x) D\left[K_{P^{*}}^{1-\alpha}[g]\right](x) d x .
$$

Corollary 3.4 (cf. [48]). Let $0<\alpha<1$. If $f_{x_{x}} I_{b}^{1-\alpha}[g] \in A C([a, b])$, then

$$
\int_{a}^{b} g(x)_{a}^{C} D_{x}^{\alpha}[f](x) d x=\left.f(x)_{x} I_{b}^{1-\alpha}[g](x)\right|_{x=a} ^{x=b}+\int_{a}^{b} f(x)_{x} D_{b}^{\alpha}[g](x) d x
$$

\section{The Generalized Fundamental Variational Problem}

By $\partial_{i} F$, we denote the partial derivative of a function $F$ with respect to its $i$ th argument. We consider the problem of finding a function $y=t \mapsto y(t), t \in[a, b]$, that gives an extremum (minimum or maximum) to the functional

$$
\partial(y)=K_{P_{1}}^{\alpha}\left[t \longmapsto F\left(t, y(t), y^{\prime}(t), B_{P_{2}}^{\beta}[y](t), K_{P_{3}}^{\gamma}[y](t)\right)\right](b)
$$

subject to the boundary conditions

$$
y(a)=y_{a}, \quad y(b)=y_{b}
$$


where $\alpha, \beta, \gamma \in(0,1), P_{1}=\langle a, b, b, 1,0\rangle$, and $P_{j}=\left\langle a, t, b, p_{j}, q_{j}\right\rangle, j=2,3$. For simplicity of notation, we introduce the operator $\{\cdot\}_{P_{2}, P_{3}}^{\beta, \gamma}$ defined by

$$
\{y\}_{P_{2}, P_{3}}^{\beta, \gamma}(t)=\left(t, y(t), y^{\prime}(t), B_{P_{2}}^{\beta}[\tau \longmapsto y(\tau)](t), K_{P_{3}}^{\gamma}[\tau \longmapsto y(\tau)](t)\right)
$$

With the new notation, one can write (4.1) simply as $2(y)=K_{P_{1}}^{\alpha}\left[F\{y\}_{P_{2}, P_{3}}^{\beta, \gamma}\right](b)$. The operator $K_{P_{1}}^{\alpha}$ has kernel $k_{\alpha}(x, t)$, and operators $B_{P_{2}}^{\beta}$ and $K_{P_{3}}^{\gamma}$ have kernels $h_{1-\beta}(t, \tau)$ and $h_{\gamma}(t, \tau)$, respectively. In the sequel, we assume the following:

(H1) Lagrangian $F \in C^{1}\left([a, b] \times \mathbb{R}^{4} ; \mathbb{R}\right)$,

(H2) functions $A_{P_{2}^{*}}^{\beta}\left[\tau \mapsto k_{\alpha}(b, \tau) \partial_{4} F\{y\}_{P_{2}, P_{3}}^{\beta, \gamma}(\tau)\right], K_{P_{3}^{*}}^{\gamma}\left[\tau \mapsto k_{\alpha}(b, \tau) \partial_{5} F\{y\}_{P_{2}, P_{3}}^{\beta, \gamma}(\tau)\right], D[t \mapsto$ $\left.\partial_{3} F\{y\}_{P_{2}, P_{3}}^{\beta, \gamma}(t) k_{\alpha}(b, t)\right]$, and $t \mapsto k_{\alpha}(b, t) \partial_{2} F\{y\}_{P_{2}, P_{3}}^{\beta_{1},}(t)$ are continuous on $(a, b)$,

(H3) functions $t \mapsto \partial_{3} F\{y\}_{P_{2}, P_{3}}^{\beta, \gamma}(t) k_{\alpha}(b, t), K_{P_{2}^{*}}^{1-\beta}\left[\tau \mapsto k_{\alpha}(b, \tau) \partial_{4} F\{y\}_{P_{2}, P_{3}}^{\beta, \gamma}(\tau)\right] \in A C([a, b])$;

(H4) kernels $k_{\alpha}(x, t), h_{1-\beta}(t, \tau)$, and $h_{\gamma}(t, \tau)$ are such that we are in conditions to use Theorems 3.1 or 3.2, and Theorem 3.3.

Definition 4.1. A function $y \in C^{1}([a, b] ; \mathbb{R})$ is said to be admissible for the fractional variational problem (4.1)-(4.2), if functions $B_{P_{2}}^{\beta}[y]$ and $K_{P_{3}}^{\gamma}[y]$ exist and are continuous on the interval $[a, b]$, and $y$ satisfies the given boundary conditions (4.2).

Theorem 4.2. If $y$ is a solution to problem (4.1)-(4.2), then y satisfies the generalized Euler-Lagrange equation

$$
\begin{aligned}
& k_{\alpha}(b, t) \partial_{2} F\{y\}_{P_{2}, P_{3}}^{\beta, r}(t)-\frac{d}{d t}\left(\partial_{3} F\{y\}_{P_{2}, P_{3}}^{\beta, r}(t) k_{\alpha}(b, t)\right) \\
& \quad-A_{P_{2}^{*}}^{\beta}\left[\tau \longmapsto k_{\alpha}(b, \tau) \partial_{4} F\{y\}_{P_{2}, P_{3}}^{\beta, \gamma}(\tau)\right](t)+K_{P_{3}^{*}}^{\gamma}\left[\tau \longmapsto k_{\alpha}(b, \tau) \partial_{5} F\{y\}_{P_{2}, P_{3}}^{\beta, \gamma}(\tau)\right](t)=0
\end{aligned}
$$

for all $t \in(a, b)$.

Proof. Suppose that $y$ is an extremizer of 2 . Consider the value of 2 at a nearby function $\widehat{y}=y+\varepsilon \eta$, where $\varepsilon \in \mathbb{R}$ is a small parameter, and $\eta \in C^{1}([a, b] ; \mathbb{R})$ is an arbitrary function with continuous $B$-op and $K$-op. We require that $\eta(a)=\eta(b)=0$. Let

$$
\begin{aligned}
\partial(\widehat{y}) & =J(\varepsilon)=K_{P_{1}}^{\alpha}\left[t \longmapsto F\left(t, \widehat{y}(t), \widehat{y}^{\prime}(t), B_{P_{2}}^{\beta}[\widehat{y}](t), K_{P_{3}}^{\gamma}[\widehat{y}](t)\right)\right](b) \\
& =\int_{a}^{b} k_{\alpha}(b, t) F\left(t, y(t)+\varepsilon \eta(t), \frac{d}{d t}(y(t)+\varepsilon \eta(t)), B_{P_{2}}^{\beta}[y+\varepsilon \eta](t), K_{P_{3}}^{\gamma}[y+\varepsilon \eta](t)\right) d t .
\end{aligned}
$$


A necessary condition for $y$ to be an extremizer is given by

$$
\begin{aligned}
\left.\frac{d J}{d \varepsilon}\right|_{\varepsilon=0}=0 \Longleftrightarrow K_{P_{1}}^{\alpha} & {\left[\partial_{2} F\{y\}_{P_{2}, P_{3}}^{\beta, \gamma} \eta+\partial_{3} F\{y\}_{P_{2}, P_{3}}^{\beta, \gamma} D[\eta]\right.} \\
& \left.+\partial_{4} F\{y\}_{P_{2}, P_{3}}^{\beta, r} B_{P_{2}}^{\beta}[\eta]+\partial_{5} F\{y\}_{P_{2}, P_{3}}^{\beta, \gamma} K_{P_{3}}^{\gamma}[\eta]\right](b)=0 \\
\Longleftrightarrow \int_{a}^{b}\left(\partial_{2} F\{y\}_{P_{2}, P_{3}}^{\beta, r}(t) \eta(t)+\partial_{3} F\{y\}_{P_{2}, P_{3}}^{\beta, \gamma}(t) \frac{d}{d t} \eta(t)\right. & \\
& \left.+\partial_{4} F\{y\}_{P_{2}, P_{3}}^{\beta, r}(t) B_{P_{2}}^{\beta}[\eta](t)+\partial_{5} F\{y\}_{P_{2}, P_{3}}^{\beta, \gamma}(t) K_{P_{3}}^{\gamma}[\eta](t)\right) k_{\alpha}(b, t) d t=0 .
\end{aligned}
$$

Using classical and generalized fractional integration by parts formulas (Theorems 3.1 or 3.2, and Theorem 3.3),

$$
\begin{aligned}
\int_{a}^{b} \partial_{3} F\{y\}_{P_{2}, P_{3}}^{\beta, \gamma}(t) k_{\alpha}(b, t) \frac{d}{d t} \eta(t) d t= & \left.\partial_{3} F\{y\}_{P_{2}, P_{3}}^{\beta, \gamma}(t) k_{\alpha}(b, t) \eta(t)\right|_{a} ^{b} \\
& -\int_{a}^{b} \frac{d}{d t}\left(\partial_{3} F\{y\}_{P_{2}, P_{3}}^{\beta, \gamma}(t) k_{\alpha}(b, t)\right) \eta(t) d t, \\
\int_{a}^{b} \partial_{4} F\{y\}_{P_{2}, P_{3}}^{\beta, \gamma}(t) k_{\alpha}(b, t) B_{P_{2}}^{\beta}[\eta](t) d t= & \left.K_{P_{2}^{*}}^{1-\beta}\left[\tau \longmapsto k_{\alpha}(b, \tau) \partial_{4} F\{y\}_{P_{2}, P_{3}}^{\beta, \gamma}(\tau)\right](t) \eta(t)\right|_{a} ^{b} \\
& -\int_{a}^{b} A_{P_{2}^{*}}^{\beta}\left[\tau \longmapsto k_{\alpha}(b, \tau) \partial_{4} F\{y\}_{P_{2}, P_{3}}^{\beta, \gamma}(\tau)\right](t) \eta(t) d t, \\
\int_{a}^{b} k_{\alpha}(b, t) \partial_{5} F\{y\}_{P_{2}, P_{3}}^{\beta, \gamma}(t) K_{P_{3}}^{\gamma}[\eta](t) d t= & \int_{a}^{b} K_{P_{3}^{*}}^{\gamma}\left[\tau \longmapsto k_{\alpha}(b, \tau) \partial_{5} F\{y\}_{P_{2}, P_{3}}^{\beta, \gamma}(\tau)\right](t) \eta(t) d t,
\end{aligned}
$$

where $P_{j}^{*}=\left\langle a, t, b, q_{j}, p_{j}\right\rangle, j=2,3$. Because $\eta(a)=\eta(b)=0,(4.6)$ simplifies to

$$
\begin{aligned}
& \int_{a}^{b}\left\{k_{\alpha}(b, t) \partial_{2} F\{y\}_{P_{2}, P_{3}}^{\beta, \gamma}(t)-\frac{d}{d t}\left(\partial_{3} F\{y\}_{P_{2}, P_{3}}^{\beta, \gamma}(t) k_{\alpha}(b, t)\right)\right. \\
& \left.\quad-A_{P_{2}^{*}}^{\beta}\left[\tau \longmapsto k_{\alpha}(b, \tau) \partial_{4} F\{y\}_{P_{2}, P_{3}}^{\beta, r}(\tau)\right](t)+K_{P_{3}^{*}}^{\gamma}\left[\tau \longmapsto k_{\alpha}(b, \tau) \partial_{5} F\{y\}_{P_{2}, P_{3}}^{\beta_{r}}(\tau)\right](t)\right\} \eta(t) d t=0 .
\end{aligned}
$$

We obtain (4.4) by application of the fundamental lemma of the calculus of variations (see, e.g., [49, Section 2.2]).

The following corollary gives an extension of the main result of [28].

Corollary 4.3. If $y$ is a solution to the problem of minimizing or maximizing

$$
\partial(y)={ }_{a} I_{b}^{\alpha}\left[t \longmapsto F\left(t, y(t), y^{\prime}(t),{ }_{a}^{C} D_{t}^{\beta}[y](t)\right)\right](b)
$$


in the class $y \in C^{1}([a, b] ; \mathbb{R})$ subject to the boundary conditions

$$
y(a)=y_{a}, \quad y(b)=y_{b},
$$

where $\alpha, \beta \in(0,1), F \in C^{1}\left([a, b] \times \mathbb{R}^{3} ; \mathbb{R}\right)$, and $\tau \mapsto(b-\tau)^{\alpha-1} \partial_{4} F\left(\tau, y(\tau), y^{\prime}(\tau),{ }_{a}^{C} D_{\tau}^{\beta}[y](\tau)\right)$ has continuous Riemann-Liouville fractional derivative ${ }_{t} D_{b}^{\beta}$, then

$$
\begin{gathered}
\partial_{2} F\left(t, y(t), y^{\prime}(t),{ }_{a}^{C} D_{t}^{\beta}[y](t)\right) \cdot(b-t)^{\alpha-1}-\frac{d}{d t}\left\{\partial_{3} F\left(t, y(t), y^{\prime}(t), \underset{a}{C} D_{t}^{\beta}[y](t)\right) \cdot(b-t)^{\alpha-1}\right\} \\
+{ }_{t} D_{b}^{\beta}\left[\tau \longmapsto(b-\tau)^{\alpha-1} \partial_{4} F\left(\tau, y(\tau), y^{\prime}(\tau),{ }_{a}^{C} D_{\tau}^{\beta}[y](\tau)\right)\right](t)=0
\end{gathered}
$$

for all $t \in(a, b)$.

Proof. Choose $k_{\alpha}(x, t)=(1 / \Gamma(\alpha))(x-t)^{\alpha-1}, h_{1-\beta}(t, \tau)=(1 / \Gamma(1-\beta))(t-\tau)^{-\beta}$, and $P_{2}=$ $\langle a, t, b, 1,0\rangle$. Then, the $K$-op, the $A$-op, and the $B$-op reduce to the left fractional integral, the left Riemann-Liouville, and the left Caputo fractional derivatives, respectively. Therefore, problem (4.9)-(4.10) is a particular case of problem (4.1)-(4.2) and (4.11) follows from (4.4) with $\partial_{5} F=0$.

The following result is the Caputo analogous to the main result of [50] done for the Riemann-Liouville fractional derivative.

Corollary 4.4. Let $\beta, \gamma \in(0,1)$. If $y$ is a solution to the problem

$$
\begin{gathered}
\int_{a}^{b} F\left(t, y(t), y^{\prime}(t){ }_{, a}^{C} D_{t}^{\beta}[y](t){ }_{a} I_{t}^{\gamma}[y](t)\right) d t \longrightarrow \text { extr, } \\
y \in C^{1}([a, b] ; \mathbb{R}), \\
y(a)=y_{a}, \quad y(b)=y_{b},
\end{gathered}
$$

then

$$
\begin{aligned}
& \partial_{2} F\left(t, y(t), y^{\prime}(t){ }_{, a}^{C} D_{t}^{\beta}[y](t),{ }_{a} I_{t}^{\gamma}[y](t)\right)-\frac{d}{d t} \partial_{3} F\left(t, y(t), y^{\prime}(t){ }_{, a}^{C} D_{t}^{\beta}[y](t),{ }_{a} I_{t}^{\gamma}[y](t)\right) \\
& +{ }_{t} D_{b}^{\beta}\left[\tau \longmapsto \partial_{4} F\left(\tau, y(\tau), y^{\prime}(\tau){ }_{{ }_{a}}{ }_{C} D_{\tau}^{\beta}[y](\tau){ }_{, a} I_{\tau}^{\gamma}[y](\tau)\right)\right](t) \\
& +{ }_{t} I_{b}^{\beta}\left[\tau \longmapsto \partial_{5} F\left(\tau, y(\tau), y^{\prime}(\tau){ }_{{ }_{a}}{ }_{D} D_{\tau}^{\beta}[y](\tau){ }_{, a} I_{\tau}^{\gamma}[y](\tau)\right)\right](t)=0
\end{aligned}
$$

holds for all $t \in(a, b)$.

Proof. The Euler-Lagrange equation (4.13) follows from (4.4) by choosing $p$-sets $P_{1}=$ $\langle a, b, b, 1,0\rangle, P_{2}=P_{3}=\langle a, t, b, 1,0\rangle$, and kernels $k_{\alpha}(x, t)=1, h_{1-\beta}(t, \tau)=(1 / \Gamma(1-\beta))(t-\tau)^{-\beta}$, and $h_{\gamma}(t, \tau)=(1 / \Gamma(\gamma))(t-\tau)^{\gamma-1}$. 
Remark 4.5. In the particular case when the Lagrangian $F$ of Corollary 4.4 does not depend on the fractional integral and the classical derivative, one obtains from (4.13) the Euler-Lagrange equation of [51].

\section{Generalized Free-Boundary Variational Problems}

Assume now that, in problem (4.1)-(4.2), the boundary conditions (4.2) are substituted by

$$
y(a) \text { is free and } y(b)=y_{b} .
$$

Theorem 5.1. If $y$ is a solution to the problem of extremizing functional (4.1) with (5.1) as boundary conditions, then $y$ satisfies the Euler-Lagrange equation (4.4). Moreover, the extra natural boundary condition

$$
\partial_{3} F\{y\}_{P_{2}, P_{3}}^{\beta_{1},}(a) k_{\alpha}(b, a)+K_{P_{2}^{*}}^{1-\beta}\left[\tau \longmapsto \partial_{4} F\{y\}_{P_{2}, P_{3}}^{\beta, \gamma}(\tau) k_{\alpha}(b, \tau)\right](a)=0
$$

holds.

Proof. Under the boundary conditions (5.1), we do not require $\eta$ in the proof of Theorem 4.2 to vanish at $t=a$. Therefore, following the proof of Theorem 4.2 , we obtain

$$
\begin{array}{rl}
\partial_{3} & F\{y\}_{P_{2}, P_{3}}^{\beta, \gamma}(a) k_{\alpha}(b, a) \eta(a)+\eta(a) K_{P_{2}^{*}}^{1-\beta}\left[\tau \longmapsto \partial_{4} F\{y\}_{P_{2}, P_{3}}^{\beta, \gamma}(\tau) k_{\alpha}(b, \tau)\right](a) \\
& +\int_{a}^{b} \eta(t)\left(\partial_{2} F\{y\}_{P_{2}, P_{3}}^{\beta_{,}}(t) k_{\alpha}(b, t)-\frac{d}{d t}\left(\partial_{3} F\{y\}_{P_{2}, P_{3}}^{\beta, r}(t) k_{\alpha}(b, t)\right)\right. \\
& \left.-A_{P_{2}^{*}}^{\beta}\left[\tau \longmapsto \partial_{4} F\{y\}_{P_{2}, P_{3}}^{\beta_{1},}(\tau) k_{\alpha}(b, \tau)\right](t)+K_{P_{3}^{*}}^{\gamma}\left[\tau \longmapsto \partial_{5} F\{y\}_{P_{2}, P_{3}}^{\beta_{3}}(\tau) k_{\alpha}(b, \tau)\right](t)\right) d t=0
\end{array}
$$

for every admissible $\eta \in C^{1}([a, b] ; \mathbb{R})$ with $\eta(b)=0$. In particular, condition (5.3) holds for those $\eta$ that fulfill $\eta(a)=0$. Hence, by the fundamental lemma of the calculus of variations, (4.4) is satisfied. Now, let us return to (5.3) and let $\eta$ again be arbitrary at point $t=a$. Inserting (4.4), we obtain the natural boundary condition (5.2).

Corollary 5.2. Let 2 be the functional given by

$$
\partial(y)={ }_{a} I_{b}^{\alpha}\left[t \longmapsto F\left(t, y(t){ }_{1}^{C} D_{t}^{\beta}[y](t)\right)\right](b)
$$

Let $y$ be a minimizer of 2 satisfying the boundary condition $y(b)=y_{b}$. Then, $y$ satisfies the EulerLagrange equation

$$
(b-t)^{\alpha-1} \partial_{2} F\left(t, y(t){ }_{a}^{C} D_{t}^{\alpha}[y](t)\right)+{ }_{t} D_{b}^{\alpha}\left[\tau \longmapsto(b-\tau)^{\alpha-1} \partial_{3} F\left(\tau, y(\tau){ }_{{ }_{a}}{ }^{C} D_{\tau}^{\beta} y(\tau)\right)\right](t)=0
$$


and the natural boundary condition

$$
{ }_{a} I_{b}^{1-\beta}\left[\tau \longmapsto(b-\tau)^{\alpha-1} \partial_{3} F\left(\tau, y(\tau){ }_{a}^{C} D_{\tau}^{\beta} y(\tau)\right)\right](a)=0 .
$$

Proof. Let functional (4.1) be such that it does not depend on the classical (integer) derivative $y^{\prime}(t)$ and on the $K$-op. If $P_{2}=\langle a, t, b, 1,0\rangle, h_{1-\beta}(t-\tau)=(1 / \Gamma(1-\beta))(t-\tau)^{-\beta}$, and $k_{\alpha}(x-$ $t)=(1 / \Gamma(\alpha))(x-t)^{\alpha-1}$, then the $B$-op reduces to the left fractional Caputo derivative and we deduce (5.5) and (5.6) from (4.4) and (5.2), respectively.

Corollary 5.3 (cf. Theorem 3.17 of [38]). Let 2 be the functional given by

$$
\partial(y)=\int_{a}^{b} F\left(t, y(t), y^{\prime}(t), B_{P_{2}}^{\beta}[y](t), K_{P_{3}}^{\gamma}[y](t)\right) d t
$$

If $y$ is a minimizer to 2 satisfying the boundary condition $y(b)=y_{b}$, then $y$ satisfies the EulerLagrange equation

$$
\partial_{2} F\{y\}_{P_{2}, P_{3}}^{\beta, r}(t)-\frac{d}{d t} \partial_{3} F\{y\}_{P_{2}, P_{3}}^{\beta, r}(t)-A_{P_{2}^{*}}^{\beta}\left[\partial_{4} F\{y\}_{P_{2}, P_{3}}^{\beta, \gamma}\right](t)+K_{P_{3}^{*}}^{\gamma}\left[\partial_{5} F\{y\}_{P_{2}, P_{3}}^{\beta, \gamma}\right](t)=0
$$

and the natural boundary condition

$$
\partial_{3} F\{y\}_{P_{2}, P_{3}}^{\beta, r}(a)+K_{P_{2}^{*}}^{1-\beta}\left[\partial_{4} F\{y\}_{P_{2}, P_{3}}^{\beta, r}\right](a)=0
$$

Proof. Choose, in the problem defined by (4.1) and (5.1), $k_{\alpha}(x, t) \equiv 1$. Then, (5.8) and (5.9) follow from (4.4) and (5.2), respectively.

\section{Generalized Isoperimetric Problems}

Let $\xi \in \mathbb{R}$. Among all functions $y:[a, b] \rightarrow \mathbb{R}$ satisfying boundary conditions

$$
y(a)=y_{a}, \quad y(b)=y_{b},
$$

and an isoperimetric constraint of the form

$$
\supset(y)=K_{P_{1}}^{\alpha}\left[G\{y\}_{P_{2}, P_{3}}^{\beta, r}\right](b)=\xi,
$$

we look for the one that extremizes (i.e., minimizes or maximizes) a functional

$$
\partial(y)=K_{P_{1}}^{\alpha}\left[F\{y\}_{P_{2}, P_{3}}^{\beta_{1}}\right](b)
$$

Operators $K_{P_{1}}^{\alpha}, B_{P_{2}}^{\beta}$, and $K_{P_{3}}^{\gamma}$, as well as function $F$, are the same as in problem (4.1)-(4.2). Moreover, we assume that functional (6.2) satisfies hypotheses (H1)-(H4). 
Definition 6.1. A function $y:[a, b] \rightarrow \mathbb{R}$ is said to be admissible for problem (6.1)-(6.3) if functions $B_{P_{2}}^{\beta}[y]$ and $K_{P_{3}}^{\gamma}[y]$ exist and are continuous on $[a, b]$, and $y$ satisfies the given boundary conditions (6.1) and the given isoperimetric constraint (6.2).

Definition 6.2. An admissible function $y \in C^{1}([a, b], \mathbb{R})$ is said to be an extremal for $\supset$ if it satisfies the Euler-Lagrange equation (4.4) associated with functional in (6.2), that is,

$$
\begin{aligned}
& k_{\alpha}(b, t) \partial_{2} G\{y\}_{P_{2}, P_{3}}^{\beta, r}(t)-\frac{d}{d t}\left(\partial_{3} G\{y\}_{P_{2}, P_{3}}^{\beta, r}(t) k_{\alpha}(b, t)\right) \\
& \quad-A_{P_{2}^{*}}^{\beta}\left[\tau \longmapsto k_{\alpha}(b, \tau) \partial_{4} G\{y\}_{P_{2}, P_{3}}^{\beta, \gamma}(\tau)\right](t)+K_{P_{3}^{*}}^{\gamma}\left[\tau \longmapsto k_{\alpha}(b, \tau) \partial_{5} G\{y\}_{P_{2}, P_{3}}^{\beta, r}(\tau)\right](t)=0,
\end{aligned}
$$

where $P_{j}^{*}=\left\langle a, t, b, q_{j}, p_{j}\right\rangle, j=2,3$, and $t \in(a, b)$.

Theorem 6.3. If $y$ is a solution to the isoperimetric problem (6.1)-(6.3) and is not an extremal for J, then there exists a real constant $\lambda$ such that

$$
\begin{aligned}
& k_{\alpha}(b, t) \partial_{2} H\{y\}_{P_{2}, P_{3}}^{\beta, \gamma}(t)-\frac{d}{d t}\left(\partial_{3} H\{y\}_{P_{2}, P_{3}}^{\beta, r}(t) k_{\alpha}(b, t)\right) \\
& \quad-A_{P_{2}^{*}}^{\beta}\left[\tau \longmapsto k_{\alpha}(b, \tau) \partial_{4} H\{y\}_{P_{2}, P_{3}}^{\beta, \gamma}(\tau)\right](t)+K_{P_{3}^{*}}^{\gamma}\left[\tau \longmapsto k_{\alpha}(b, \tau) \partial_{5} H\{y\}_{P_{2}, P_{3}}^{\beta, \gamma}(\tau)\right](t)=0
\end{aligned}
$$

for all $t \in(a, b)$, where $H(t, y, u, v, w)=F(t, y, u, v, w)-\lambda G(t, y, u, v, w)$ and $P_{j}^{*}=\left\langle a, t, b, q_{j}, p_{j}\right\rangle$, $j=2,3$.

Proof. Consider a two-parameter family of the form $\hat{y}=y+\varepsilon_{1} \eta_{1}+\varepsilon_{2} \eta_{2}$, where, for each $i \in\{1,2\}$, we have $\eta_{i}(a)=\eta_{i}(b)=0$. First, we show that we can select $\varepsilon_{2} \eta_{2}$ such that $\hat{y}$ satisfies (6.2). Consider the quantity $\supset(\widehat{y})=K_{P_{1}}^{\alpha}\left[G\{\widehat{y}\}_{P_{2}, P_{3}}^{\beta, \gamma}\right](b)$. Looking to $\supset(\widehat{y})$ as a function of $\varepsilon_{1}, \varepsilon_{2}$, we define $\widehat{I}\left(\varepsilon_{1}, \varepsilon_{2}\right)=\supset(\widehat{y})-\xi$. Thus, $\widehat{I}(0,0)=0$. On the other hand, applying integration by parts formulas (Theorems 3.1 or 3.2, and Theorem 3.3), we obtain that

$$
\begin{aligned}
\left.\frac{\partial \widehat{I}}{\partial \varepsilon_{2}}\right|_{(0,0)}=\int_{a}^{b} \eta_{2}(t)( & k_{\alpha}(b, t) \partial_{2} G\{y\}_{P_{2}, P_{3}}^{\beta, r}(t)-\frac{d}{d t}\left(\partial_{3} G\{y\}_{P_{2}, P_{3}}^{\beta, r}(t) k_{\alpha}(b, t)\right) \\
& -A_{P_{2}^{*}}^{\beta}\left[\tau \longmapsto k_{\alpha}(b, \tau) \partial_{4} G\{y\}_{P_{2}, P_{3}}^{\beta, r}(\tau)\right](t) \\
& \left.+K_{P_{3}^{*}}^{\gamma}\left[\tau \longmapsto k_{\alpha}(b, \tau) \partial_{5} G\{y\}_{P_{2}, P_{3}}^{\beta_{\gamma}}(\tau)\right](t)\right) d t,
\end{aligned}
$$

where $P_{j}^{*}=\left\langle a, t, b, q_{j}, p_{j}\right\rangle, j=2,3$. We assume that $y$ is not an extremal for $\supset$. Hence, the fundamental lemma of the calculus of variations implies that there exists a function $\eta_{2}$ such that $\partial \widehat{I} /\left.\partial \varepsilon_{2}\right|_{(0,0)} \neq 0$. According to the implicit function theorem, there exists a function $\varepsilon_{2}(\cdot)$ defined in a neighborhood of 0 such that $\widehat{I}\left(\varepsilon_{1}, \varepsilon_{2}\left(\varepsilon_{1}\right)\right)=0$. Let $\widehat{J}\left(\varepsilon_{1}, \varepsilon_{2}\right)=2(\widehat{y})$. Function $\widehat{J}$ has an extremum at $(0,0)$ subject to $\widehat{I}(0,0)=0$, and we have proved that 
$\nabla \widehat{I}(0,0) \neq 0$. The Lagrange multiplier rule asserts that there exists a real number $\lambda$ such that $\nabla(\widehat{J}(0,0)-\lambda \widehat{I}(0,0))=0$. Because

$$
\begin{aligned}
& \left.\frac{\partial \widehat{J}}{\partial \varepsilon_{1}}\right|_{(0,0)}=\int_{a}^{b}\left(k_{\alpha}(b, t) \partial_{2} F\{y\}_{P_{2}, P_{3}}^{\beta_{\gamma},}(t)-\frac{d}{d t}\left(\partial_{3} F\{y\}_{P_{2}, P_{3}}^{\beta_{,},}(t) k_{\alpha}(b, t)\right)\right. \\
& -A_{P_{2}^{*}}^{\beta}\left[\tau \longmapsto k_{\alpha}(b, \tau) \partial_{4} F\{y\}_{P_{2}, P_{3}}^{\beta, \gamma}(\tau)\right](t) \\
& \left.+K_{P_{3}^{*}}^{\gamma}\left[\tau \longmapsto k_{\alpha}(b, \tau) \partial_{5} F\{y\}_{P_{2}, P_{3}}^{\beta, \gamma}(\tau)\right](t)\right) \eta_{1}(t) d t, \\
& \left.\frac{\partial \widehat{I}}{\partial \varepsilon_{1}}\right|_{(0,0)}=\int_{a}^{b}\left(k_{\alpha}(b, t) \partial_{2} G\{y\}_{P_{2}, P_{3}}^{\beta_{\gamma},}(t)-\frac{d}{d t}\left(\partial_{3} G\{y\}_{P_{2}, P_{3}}^{\beta_{\gamma},}(t) k_{\alpha}(b, t)\right)\right. \\
& -A_{P_{2}^{*}}^{\beta}\left[\tau \longmapsto k_{\alpha}(b, \tau) \partial_{4} G\{y\}_{P_{2}, P_{3}}^{\beta, \gamma}(\tau)\right](t) \\
& \left.+K_{P_{3}^{*}}^{\gamma}\left[\tau \longmapsto k_{\alpha}(b, \tau) \partial_{5} G\{y\}_{P_{2}, P_{3}}^{\beta, \gamma}(\tau)\right](t)\right) \eta_{1}(t) d t,
\end{aligned}
$$

one has

$$
\begin{aligned}
& \int_{a}^{b}\left\{k_{\alpha}(b, t) \partial_{2} F\{y\}_{P_{2}, P_{3}}^{\beta, \gamma}(t)-\frac{d}{d t}\left(\partial_{3} F\{y\}_{P_{2}, P_{3}}^{\beta, r}(t) k_{\alpha}(b, t)\right)\right. \\
& -A_{P_{2}^{*}}^{\beta}\left[\tau \longmapsto k_{\alpha}(b, \tau) \partial_{4} F\{y\}_{P_{2}, P_{3}}^{\beta, \gamma}(\tau)\right](t)+K_{P_{3}^{*}}^{\gamma}\left[\tau \longmapsto k_{\alpha}(b, \tau) \partial_{5} F\{y\}_{P_{2}, P_{3}}^{\beta_{3}}(\tau)\right](t) \\
& -\lambda\left(k_{\alpha}(b, t) \partial_{2} G\{y\}_{P_{2}, P_{3}}^{\beta, \gamma}(t)-\frac{d}{d t}\left(\partial_{3} G\{y\}_{P_{2}, P_{3}}^{\beta_{3}}(t) k_{\alpha}(b, t)\right)\right. \\
& -A_{P_{2}^{*}}^{\beta}\left[\tau \longmapsto k_{\alpha}(b, \tau) \partial_{4} G\{y\}_{P_{2}, P_{3}}^{\beta, \gamma}(\tau)\right](t) \\
& \left.\left.+K_{P_{3}^{*}}^{\gamma}\left[\tau \longmapsto k_{\alpha}(b, \tau) \partial_{5} G\{y\}_{P_{2}, P_{3}}^{\beta, \gamma}(\tau)\right](t)\right)\right\} \eta_{1}(t) d t=0 .
\end{aligned}
$$

From the fundamental lemma of the calculus of variations (see, e.g., [49], Section 2.2), it follows

$$
\begin{aligned}
k_{\alpha}(b, t) \partial_{2} F\{y\}_{P_{2}, P_{3}}^{\beta, \gamma}(t)-\frac{d}{d t}\left(\partial_{3} F\{y\}_{P_{2}, P_{3}}^{\beta, \gamma}(t) k_{\alpha}(b, t)\right) \\
\quad-A_{P_{2}^{*}}^{\beta}\left[\tau \longmapsto k_{\alpha}(b, \tau) \partial_{4} F\{y\}_{P_{2}, P_{3}}^{\beta_{1} \gamma}(\tau)\right](t)+K_{P_{3}^{*}}^{\gamma}\left[\tau \longmapsto k_{\alpha}(b, \tau) \partial_{5} F\{y\}_{P_{2}, P_{3}}^{\beta_{1} \gamma}(\tau)\right](t) \\
\quad-\lambda\left(k_{\alpha}(b, t) \partial_{2} G\{y\}_{P_{2}, P_{3}}^{\beta_{3},}(t)-\frac{d}{d t}\left(\partial_{3} G\{y\}_{P_{2}, P_{3}}^{\beta_{3},}(t) k_{\alpha}(b, t)\right)\right. \\
\left.-A_{P_{2}^{*}}^{\beta}\left[\tau \longmapsto k_{\alpha}(b, \tau) \partial_{4} G\{y\}_{P_{2}, P_{3}}^{\beta, \gamma}(\tau)\right](t)+K_{P_{3}^{*}}^{\gamma}\left[\tau \longmapsto k_{\alpha}(b, \tau) \partial_{5} G\{y\}_{P_{2}, P_{3}}^{\beta, \gamma}(\tau)\right](t)\right)=0,
\end{aligned}
$$


that is,

$$
\begin{aligned}
& k_{\alpha}(b, t) \partial_{2} H\{y\}_{P_{2}, P_{3}}^{\beta, \gamma}(t)-\frac{d}{d t}\left(\partial_{3} H\{y\}_{P_{2}, P_{3}}^{\beta, r}(t) k_{\alpha}(b, t)\right) \\
& -A_{P_{2}^{*}}^{\beta}\left[\tau \longmapsto k_{\alpha}(b, \tau) \partial_{4} H\{y\}_{P_{2}, P_{3}}^{\beta, \gamma}(\tau)\right](t)+K_{P_{3}^{*}}^{\gamma}\left[\tau \longmapsto k_{\alpha}(b, \tau) \partial_{5} H\{y\}_{P_{2}, P_{3}}^{\beta, \gamma}(\tau)\right](t)=0
\end{aligned}
$$

with $H=F-\lambda G$.

Corollary 6.4. Let $y$ be a minimizer to the isoperimetric problem

$$
\begin{gathered}
\partial(y)={ }_{a} I_{b}^{\alpha}\left[t \longmapsto F\left(t, y(t){ }_{, a}^{C} D_{t}^{\beta}[y](t)\right)\right](b) \longrightarrow \min , \\
\mathcal{O}(y)={ }_{a} I_{b}^{\alpha}\left[t \longmapsto G\left(t, y(t){ }_{, a}^{C} D_{t}^{\beta}[y](t)\right)\right](b)=\xi, \\
y(a)=y_{a}, \quad y(b)=y_{b} .
\end{gathered}
$$

If $y$ is not an extremal of $\mathcal{O}$, then there exists a constant $\lambda$ such that $y$ satisfies

$$
(b-t)^{\alpha-1} \partial_{2} H\left(t, y(t){ }_{, a}^{C} D_{t}^{\alpha}[y](t)\right)+{ }_{t} D_{b}^{\beta}\left[\tau \longmapsto(b-\tau)^{\alpha-1} \partial_{3} H\left(\tau, y(\tau){ }_{, a}^{C} D_{\tau}^{\beta}[y](\tau)\right)\right](t)=0
$$

for all $t \in(a, b)$, where $H(t, y, v)=F(t, y, v)-\lambda G(t, y, v)$.

Proof. Let $k_{\alpha}(x, t)=(1 / \Gamma(\alpha))(x-t)^{\alpha-1}, h_{1-\beta}(t, \tau)=(1 / \Gamma(1-\beta))(t-\tau)^{-\beta}, P_{1}=\langle a, b, b, 1,0\rangle$, and $P_{2}=\langle a, t, b, 1,0\rangle$. Then, the $K$-op and the $B$-op reduce to the left fractional integral and the left fractional Caputo derivative, respectively. Therefore, problem (6.11) is a particular case of problem (6.1)-(6.3), and (6.12) follows from (6.5) with $\partial_{3} H=\partial_{5} H=0$.

Corollary 6.5 (cf. Theorem 3.22 of [38]). Let $y$ be a minimizer to

$$
\begin{gathered}
\partial(y)=\int_{a}^{b} F\left(t, y(t), y^{\prime}(t), B_{P_{2}}^{\beta}[y](t), K_{P_{3}}^{\gamma}[y](t)\right) d t \rightarrow \min , \\
\partial(y)=\int_{a}^{b} G\left(t, y(t), y^{\prime}(t), B_{P_{2}}^{\beta}[y](t), K_{P_{3}}^{\gamma}[y](t)\right) d t=\xi, \\
y(a)=y_{a}, \quad y(b)=y_{b} .
\end{gathered}
$$

If $y$ is not an extremal of $\mathcal{O}$, then there exists a constant $\lambda$ such that $y$ satisfies

$$
\partial_{2} H\{y\}_{P_{2}, P_{3}}^{\beta_{r},}(t)-\frac{d}{d t} \partial_{3} H\{y\}_{P_{2}, P_{3}}^{\beta, \gamma}(t)-A_{P_{2}^{*}}^{\beta}\left[\partial_{4} H\{y\}_{P_{2}, P_{3}}^{\beta, r}\right](t)+K_{P_{3}^{*}}^{\gamma}\left[\partial_{5} H\{y\}_{P_{2}, P_{3}}^{\beta, r}\right](t)=0
$$

for all $t \in(a, b)$, where $H(t, y, u, v, w)=F(t, y, u, v, w)-\lambda G(t, y, u, v, w)$. 
Proof. Let, in problem (6.1)-(6.3), $P_{1}=\langle a, b, b, 1,0\rangle$ and kernel $k_{\alpha}(x, t) \equiv 1$. Then, the generalized fractional integral $K_{P_{1}}^{\alpha}$ becomes the classical integral and (6.14) follows from (6.5).

\section{Illustrative Examples}

We illustrate our results through two examples with different kernels: one of a fundamental problem (4.1)-(4.2) (Example 7.1), the other an isoperimetric problem (6.1)-(6.3) (Example 7.2).

Example 7.1. Let $\alpha, \beta \in(0,1), \xi \in \mathbb{R}, P_{1}=\langle 0,1,1,1,0\rangle$, and $P_{2}=\langle 0, t, 1,1,0\rangle$. Consider the following problem:

$$
\begin{gathered}
\partial(y)=K_{P_{1}}^{\alpha}\left[t \longmapsto t K_{P_{2}}^{\beta}[y](t)+\sqrt{1-\left(K_{P_{2}}^{\beta}[y](t)\right)^{2}}\right](1) \longrightarrow \min , \\
y(0)=1, \quad y(1)=\frac{\sqrt{2}}{4}+\int_{0}^{1} r_{\beta}(1-\tau) \frac{1}{\left(1+\tau^{2}\right)^{3 / 2}} d \tau,
\end{gathered}
$$

with kernel $h_{\beta}$ such that $h_{\beta}(t, \tau)=h_{\beta}(t-\tau)$ and $h_{\beta}(0)=1$. Here, the resolvent $r_{\beta}(t)$ is related to the kernel $h_{\beta}(t)$ by $r_{\beta}(t)=\mathcal{L}^{-1}\left[s \mapsto\left(1 / s \tilde{h}_{\beta}(s)\right)-1\right](t), \tilde{h}_{\beta}(s)=\mathcal{L}\left[t \mapsto h_{\beta}(t)\right](s)$, where $\mathcal{L}$ and $\mathcal{L}^{-1}$ are the direct and the inverse Laplace operators, respectively. We apply Theorem 4.2 with Lagrangian $F$ given by $F(t, y, u, v, w)=t w+\sqrt{1-w^{2}}$. Because

$$
y(t)=\frac{1}{\left(1+t^{2}\right)^{3 / 2}}+\int_{0}^{t} r_{\beta}(t-\tau) \frac{1}{\left(1+\tau^{2}\right)^{3 / 2}} d \tau
$$

is the solution to the Volterra integral equation of first kind (see, e.g., Equation 16, page 114 of [47])

$$
K_{P_{2}}^{\beta}[y](t)=\frac{t \sqrt{1+t^{2}}}{1+t^{2}}
$$

it satisfies our generalized Euler-Lagrange equation (4.4), that is,

$$
K_{P_{2}^{*}}^{\beta}\left[\tau \longmapsto k_{\alpha}(b, \tau)\left(\frac{-K_{P_{2}^{*}}^{\beta}[y](\tau)}{\sqrt{1-\left(K_{P_{2}^{*}}^{\beta}[y](\tau)\right)^{2}}}+\tau\right)\right](t)=0 .
$$

In particular, for the kernel $h_{\beta}(t-\tau)=\cosh (\beta(t-\tau))$, the boundary conditions are $y(0)=1$ and $y(1)=1+\beta^{2}(1-\sqrt{2})$, and the solution is $y(t)=\left(1 /\left(1+t^{2}\right)^{3 / 2}\right)+\beta^{2}\left(1-\sqrt{1+t^{2}}\right)$ (cf. [47], page 22). 
In the next example, we make use of the Mittag-Leffler function of two parameters: if $\alpha, \beta>0$, then the Mittag-Leffler function is defined by

$$
E_{\alpha, \beta}(z)=\sum_{k=0}^{\infty} \frac{z^{k}}{\Gamma(\alpha k+\beta)}
$$

This function appears naturally in the solution of fractional differential equations, as a generalization of the exponential function [52].

Example 7.2. Let $\alpha, \beta \in(0,1), \xi \in \mathbb{R}$, and $\xi \notin\{ \pm 1 / 4\}$. Consider the following problem:

$$
\begin{gathered}
\partial(y)={ }_{0} I_{1}^{\alpha}\left[\sqrt{1+\left(y^{\prime}+{ }_{0}^{C} D_{t}^{\beta}[y]\right)^{2}}\right](1) \longrightarrow \min , \\
\supset(y)={ }_{0} I_{1}^{\alpha}\left[\left(y^{\prime}+{ }_{0}^{C} D_{t}^{\beta}[y]\right)^{2}\right](1)=\xi, \\
y(0)=0, \quad y(1)=\int_{0}^{1} E_{1-\beta, 1}\left(-(1-\tau)^{1-\beta}\right) \frac{\sqrt{1-16 \xi^{2}}}{4 \xi} d \tau,
\end{gathered}
$$

which is an example of (6.1)-(6.3) with $p$-sets $P_{1}=\langle 0,1,1,1,0\rangle$ and $P_{2}=\langle 0, t, 1,1,0\rangle$ and kernels $k_{\alpha}(x-t)=(1 / \Gamma(\alpha))(x-t)^{\alpha-1}$ and $h_{1-\beta}(t-\tau)=(1 / \Gamma(1-\beta))(t-\tau)^{-\beta}$. Function $H$ of Theorem 6.3 is given by $H(t, y, u, v, w)=\sqrt{1+(u+v)^{2}}-\lambda(u+v)^{2}$. One can easily check (see [52], page 324) that

$$
y(t)=\int_{0}^{t} E_{1-\beta, 1}\left(-(t-\tau)^{1-\beta}\right) \frac{\sqrt{1-16 \xi^{2}}}{4 \xi} d \tau
$$

(i) is not an extremal for $\bigcirc$,

(ii) satisfies $y^{\prime}+{ }_{0}^{C} D_{t}^{\beta}[y]=\sqrt{1-16 \xi^{2}} / 4 \xi$.

Moreover, (7.7) satisfies (6.5) for $\lambda=2 \xi$, that is,

$$
\begin{aligned}
& -\frac{d}{d t}\left((1-t)^{\alpha-1}\left(y^{\prime}(t)+{ }_{0}^{C} D_{t}^{\beta}[y](t)\right)\left(\frac{1}{\sqrt{1+\left(y^{\prime}(t)+{ }_{0}^{C} D_{t}^{\beta}[y](t)\right)^{2}}}-4 \xi\right)\right) \\
& +{ }_{t} D_{1}^{\beta}\left[\tau \longmapsto(1-\tau)^{\alpha-1}\left(y^{\prime}(\tau)+{ }_{0}^{C} D_{\tau}^{\beta}[y](\tau)\right)\left(\frac{1}{\sqrt{1+\left(y^{\prime}(\tau)+{ }_{0}^{C} D_{\tau}^{\beta}[y](\tau)\right)^{2}}}-4 \xi\right)\right](t)=0
\end{aligned}
$$

for all $t \in(0,1)$. We conclude that (7.7) is an extremal for problem (7.6). 


\section{Applications to Physics}

If the functional (4.1) does not depend on $B$-op and $K$-op, then Theorem 4.2 gives the following result: if $y$ is a solution to the problem of extremizing

$$
\partial(y)=\int_{a}^{b} F\left(t, y(t), y^{\prime}(t)\right) k_{\alpha}(b, t) d t
$$

subject to $y(a)=y_{a}$ and $y(b)=y_{b}$, where $\alpha \in(0,1)$, then

$$
\partial_{2} F\left(t, y(t), y^{\prime}(t)\right)-\frac{d}{d t} \partial_{3} F\left(t, y(t), y^{\prime}(t)\right)=\frac{1}{k_{\alpha}(b, t)} \cdot \frac{d}{d t} k_{\alpha}(b, t) \partial_{3} F\left(t, y(t), y^{\prime}(t)\right) .
$$

We recognize on the right hand side of (8.2) the generalized weak dissipative parameter

$$
\delta(t)=\frac{1}{k_{\alpha}(b, t)} \cdot \frac{d}{d t} k_{\alpha}(b, t)
$$

\subsection{Quantum Mechanics of the Damped Harmonic Oscillator}

As a first application, let us consider kernel $k_{\alpha}(b, t)=\mathrm{e}^{\alpha(b-t)}$ and the Lagrangian

$$
L(y, \dot{y})=\frac{1}{2} m \dot{y}^{2}-V(y)
$$

where $V(y)$ is the potential energy and $m$ stands for mass. The Euler-Lagrange equation (8.2) gives the following second-order ordinary differential equation:

$$
\ddot{y}(t)-\alpha \dot{y}(t)=-\frac{1}{m} V^{\prime}(y(t)) .
$$

Equation (8.5) coincides with (14) of [44], obtained by modification of Hamilton's principle.

\subsection{Fractional Action-Like Variational Approach (FALVA)}

We now extend some of the recent results of [39, 41-43], where the fractional actionlike variational approach (FALVA) was proposed to model dynamical systems. FALVA functionals are particular cases of (8.1), where the fractional time integral introduces only one parameter $\alpha$. Let us consider the Caldirola-Kanai Lagrangian [39, 40, 42]

$$
L(t, y, \dot{y})=m(t)\left(\frac{\dot{y}^{2}}{2}-\omega^{2} \frac{y^{2}}{2}\right)
$$

which describes a dynamical oscillatory system with exponentially increasing time-dependent mass, where $\omega$ is the frequency and $m(t)=m_{0} \mathrm{e}^{-\gamma b} \mathrm{e}^{\gamma t}=\bar{m}_{0} \mathrm{e}^{\gamma t}, \bar{m}_{0}=m_{0} \mathrm{e}^{-\gamma b}$. Using our 
generalized FALVA Euler-Lagrange equation (8.2) with kernel $k_{\alpha}(b, t)$ to Lagrangian (8.6), we obtain

$$
\ddot{y}(t)+(\delta(t)+\gamma) \dot{y}(t)+\omega^{2} y(t)=0
$$

We study two particular kernels.

(1) If we choose kernel

$$
k_{\alpha}(b, t)=\frac{(\rho+1)^{1-\alpha}}{\Gamma(\alpha)}\left(b^{\rho+1}-t^{\rho+1}\right) t^{\rho}
$$

defined in [53], then the Euler-Lagrange equation is

$$
\partial_{2} F\left(t, y(t), y^{\prime}(t)\right)-\frac{d}{d t} \partial_{3} F\left(t, y(t), y^{\prime}(t)\right)=\left(\frac{(1-\alpha)(\rho+1) t^{\rho}}{b^{\rho+1}-t^{\rho+1}}\right) \partial_{3} F\left(t, y(t), y^{\prime}(t)\right)
$$

In particular, when $\rho \rightarrow 0$, (8.8) becomes the kernel of the Riemann-Liouville fractional integral, and equation (8.9) gives

$$
\partial_{2} F\left(t, y(t), y^{\prime}(t)\right)-\frac{d}{d t} \partial_{3} F\left(t, y(t), y^{\prime}(t)\right)=\frac{1-\alpha}{b-t} \partial_{3} F\left(t, y(t), y^{\prime}(t)\right)
$$

which is the Euler-Lagrange equation proved in [42]. For $\rho \neq 0$, we have

$$
\delta(t)=\frac{(1-\alpha)(\rho+1) t^{\rho}}{b^{\rho+1}-t^{\rho+1}} \longrightarrow 0, \quad \text { if } t \longrightarrow \infty \text { or } t \longrightarrow 0
$$

Therefore, both at the very early time and at very large time, dissipation disappears. Moreover, if $\rho \rightarrow 0$, then

$$
\delta(t)=\frac{1-\alpha}{b-t} \longrightarrow \begin{cases}0, & \text { if } t \longrightarrow \infty \\ \frac{1-\alpha}{b}, & \text { if } t \longrightarrow 0\end{cases}
$$

This shows that, at the origin of time, the time-dependent dissipation becomes stationary and that, at very large time, no dissipation, of any kind, exists.

(2) If we choose kernel $k_{\alpha}(b, t)=(\cosh b-\cosh t)^{\alpha-1}$, then

$$
\begin{gathered}
\partial_{2} F\left(t, y(t), y^{\prime}(t)\right)-\frac{d}{d t} \partial_{3} F\left(t, y(t), y^{\prime}(t)\right)=-(\alpha-1) \frac{\sinh t}{\cosh b-\cosh t} \partial_{3} F\left(t, y(t), y^{\prime}(t)\right), \\
\delta(t)=-(\alpha-1) \frac{\sinh t}{\cosh b-\cosh t} \longrightarrow \begin{cases}\alpha-1, & \text { if } t \longrightarrow \infty, \\
0, & \text { if } t \longrightarrow 0 .\end{cases}
\end{gathered}
$$


In contrast with previous case, item 1, here dissipation does not disappear at latetime dynamics.

We note that there is a small inconsistence in [42], regarding the coefficient of $\dot{y}(t)$ in (8.7), and a small inconsistence in [39], regarding a sign of (8.13).

\section{Conclusion}

In this paper, we unify, subsume, and significantly extend the necessary optimality conditions available in the literature of the fractional calculus of variations. It should be mentioned, however, that since fractional operators are nonlocal, it can be extremely challenging to find analytical solutions to fractional problems of the calculus of variations, and, in many cases, solutions may not exist. In our paper, we give two examples with analytic solutions, and many more can be found borrowing different kernels from [47]. On the other hand, one can easily choose examples for which the fractional Euler-Lagrange differential equations are hard to solve, and, in that case, one needs to use numerical methods [54-57]. The question of existence of solutions to fractional variational problems is a complete open area of research. This needs attention. Indeed, in the absence of existence, the necessary conditions for extremality are vacuous: one cannot characterize an entity that does not exist in the first place. For solving a problem of the fractional calculus of variations, one should proceed along the following three steps: (i) first, prove that a solution to the problem exists; (ii) second, verify the applicability of necessary optimality conditions; (iii) finally, apply the necessary conditions which identify the extremals (the candidates). Further elimination, if necessary, identifies the minimizer(s) of the problem. All three steps in the above procedure are crucial. As mentioned by Young in [58], the calculus of variations has born from the study of necessary optimality conditions, but any such theory is "naive" until the existence of minimizers is verified. The process leading to the existence theorems was introduced by Tonelli in 1915 by the so-called direct method [59]. During two centuries, mathematicians were developing "the naive approach to the calculus of variations". There were, of course, good reasons why the existence problem was only solved in the beginning of XX century, two hundred years after necessary optimality conditions began to be studied: see $[60,61]$ and references therein. Similar situation happens now with the fractional calculus of variations: the subject is only fifteen years old and is still in the "naive period". We believe time has come to address the existence question, and this will be considered in a forthcoming paper.

\section{Acknowledgments}

This work was supported by FEDER funds through COMPETE-Operational Programme Factors of Competitiveness (Programa Operacional Factores de Competitividade) and by Portuguese funds through the Center for Research and Development in Mathematics and Applications (University of Aveiro) and the Portuguese Foundation for Science and Technology (FCT-Fundação para a Ciência e a Tecnologia), within project PEst-C/MAT/UI4106/2011 with COMPETE no. FCOMP-01-0124-FEDER-022690. T. Odzijewicz was also supported by FCT through the Ph.D. fellowship SFRH/BD/33865/2009; A. B. Malinowska by Białystok University of Technology Grant S/WI/02/2011; D. F. M. Torres by FCT through the Portugal-Austin (USA) cooperation project UTAustin/MAT/0057/2008. This paper is part of 
the first author's Ph.D., which is carried out at the University of Aveiro under the Doctoral Programme Mathematics and Applications of Universities of Aveiro and Minho.

\section{References}

[1] B. S. Mordukhovich, Variational Analysis and Generalized Differentiation. I, vol. 330 of Grundlehren der Mathematischen Wissenschaften, Springer, Berlin, Germany, 2006.

[2] B. S. Mordukhovich, Variational Analysis and Generalized Differentiation. II, vol. 331 of Grundlehren der Mathematischen Wissenschaften, Springer, Berlin, Germany, 2006.

[3] B. van Brunt, The Calculus of Variations, Universitext, Springer, New York, NY, USA, 2004.

[4] A. B. Malinowska and D. F. M. Torres, "Nonessential functionals in multiobjective optimal control problems," Proceedings of the Estonian Academy of Sciences. Physics, Mathematics, vol. 56, no. 4, pp. 336346, 2007.

[5] N. Martins and D. F. M. Torres, "Calculus of variations on time scales with nabla derivatives," Nonlinear Analysis: Theory, Methods E Applications, vol. 71, no. 12, pp. e763-e773, 2009.

[6] T. Odzijewicz and D. F. M. Torres, "Fractional calculus of variations for double integrals," Balkan Journal of Geometry and Its Applications, vol. 16, no. 2, pp. 102-113, 2011.

[7] F. Riewe, "Nonconservative Lagrangian and Hamiltonian mechanics," Physical Review E, vol. 53, no. 2, pp. 1890-1899, 1996.

[8] F. Riewe, "Mechanics with fractional derivatives," Physical Review E, vol. 55, no. 3, pp. 3581-3592, 1997.

[9] K. Balachandran, J. Y. Park, and J. J. Trujillo, “Controllability of nonlinear fractional dynamical systems," Nonlinear Analysis: Theory, Methods E Applications, vol. 75, no. 4, pp. 1919-1926, 2012.

[10] I. Podlubny, Fractional Differential Equations, vol. 198 of Mathematics in Science and Engineering, Academic Press, San Diego, Calif, USA, 1999.

[11] R. Metzler and J. Klafter, "The random walk's guide to anomalous diffusion: a fractional dynamics approach," Physics Reports, vol. 339, no. 1, p. 77, 2000.

[12] G. M. Zaslavsky, Hamiltonian Chaos and Fractional Dynamics, Oxford University Press, Oxford, UK, 2008.

[13] F. Mainardi, Fractional Calculus and Waves in Linear Viscoelasticity, Imperial College Press, London, UK, 2010.

[14] A. Carpinteri and F. Mainardi, Fractals and Fractional Calculus in Continuum Mechanics, vol. 378 of CISM Courses and Lectures, Springer, Vienna, Austria, 1997.

[15] J. Li and M. Ostoja-Starzewski, "Micropolar continuum mechanics of fractal media," International Journal of Engineering Science, vol. 49, no. 12, pp. 1302-1310, 2011.

[16] R. Hilfer, Applications of Fractional Calculus in Physics, World Scientific, River Edge, NJ, USA, 2000.

[17] N. Laskin, "Fractional quantum mechanics and Lévy path integrals," Physics Letters A, vol. 268, no. 4-6, pp. 298-305, 2000.

[18] G. M. Zaslavsky and M. A. Edelman, "Fractional kinetics: From pseudochaotic dynamics to Maxwell's Demon," Physica D, vol. 193, no. 1-4, pp. 128-147, 2004.

[19] V. E. Tarasov, "Fractional statistical mechanics," Chaos, vol. 16, no. 3, Article ID 033108, 7 pages, 2006.

[20] V. E. Tarasov, "Continuous limit of discrete systems with long-range interaction," Journal of Physics A, vol. 39, no. 48, pp. 14895-14910, 2006.

[21] V. E. Tarasov and G. M. Zaslavsky, "Fractional dynamics of coupled oscillators with long-range interaction," Chaos, vol. 16, no. 2, Article ID 023110, 13 pages, 2006.

[22] C. Lánczos, The Variational Principles of Pechanics, Mathematical Expositions, No. 4, University of Toronto Press, Toronto, Canada, 4th edition, 1970.

[23] R. Almeida, "Fractional variational problems with the Riesz-Caputo derivative," Applied Mathematics Letters, vol. 25, no. 2, pp. 142-148, 2012.

[24] R. Almeida, A. B. Malinowska, and D. F. M. Torres, "A fractional calculus of variations for multiple integrals with application to vibrating string," Journal of Mathematical Physics, vol. 51, no. 3, Article ID 033503, 12 pages, 2010.

[25] N. R. O. Bastos, R. A. C. Ferreira, and D. F. M. Torres, "Necessary optimality conditions for fractional difference problems of the calculus of variations," Discrete and Continuous Dynamical Systems. Series A, vol. 29, no. 2, pp. 417-437, 2011.

[26] N. R. O. Bastos, R. A. C. Ferreira, and D. F. M. Torres, "Discrete-time fractional variational problems," Signal Process, vol. 91, no. 3, pp. 513-524, 2011. 
[27] J. Cresson, "Fractional embedding of differential operators and Lagrangian systems," Journal of Mathematical Physics, vol. 48, no. 3, Article ID 033504, 34 pages, 2007.

[28] R. A. El-Nabulsi and D. F. M. Torres, "Fractional actionlike variational problems," Journal of Mathematical Physics, vol. 49, no. 5, Article ID 053521, 7 pages, 2008.

[29] G. S. F. Frederico and D. F. M. Torres, "Fractional conservation laws in optimal control theory," Nonlinear Dynamics, vol. 53, no. 3, pp. 215-222, 2008.

[30] A. B. Malinowska, "Fractional variational calculus for non-differentiable functions," in Fractional Dynamics and Control, D. Baleanu, J. A. Tenreiro Machado, and A. C. J. Luo, Eds., part 2, chapter 8, pp. 97-108, Springer, New York, NY, USA, 2012.

[31] A. B. Malinowska and D. F. M. Torres, "Generalized natural boundary conditions for fractional variational problems in terms of the Caputo derivative," Computers $\mathcal{E}$ Mathematics with Applications, vol. 59, no. 9, pp. 3110-3116, 2010.

[32] D. Mozyrska and D. F. M. Torres, "Minimal modified energy control for fractional linear control systems with the Caputo derivative," Carpathian Journal of Mathematics, vol. 26, no. 2, pp. 210-221, 2010.

[33] D. Mozyrska and D. F. M. Torres, "Modified optimal energy and initial memory of fractional continuous-time linear systems," Signal Processing, vol. 91, no. 3, pp. 379-385, 2011.

[34] T. Odzijewicz, A. B. Malinowska, and D. F. M. Torres, "Fractional variational calculus with classical and combined Caputo derivatives," Nonlinear Analysis: Theory, Methods and Applications, vol. 75, no. 3, pp. 1507-1515, 2012.

[35] Z. Sha, F. Jing-Li, and L. Yong-Song, "Lagrange equations of nonholonomic systems with fractional derivatives," Chinese Physics B, vol. 19, no. 12, Article ID 120301, 2010.

[36] O. P. Agrawal, "Generalized variational problems and Euler-Lagrange equations," Computers $\mathcal{E}$ Mathematics with Applications, vol. 59, no. 5, pp. 1852-1864, 2010.

[37] V. Kiryakova, Generalized Fractional Calculus and Applications, vol. 301 of Pitman Research Notes in Mathematics Series, Longman Scientific \& Technical, Harlow, UK, 1994.

[38] T. Odzijewicz, A. B. Malinowska, and D. F. M. Torres, "Generalized fractional calculus with applications to the calculus of variations," Computers $\mathcal{E}$ Mathematics with Applications. In press.

[39] A. R. El-Nabulsi, "Fractional variational problems from extended exponentially fractional integral," Applied Mathematics and Computation, vol. 217, no. 22, pp. 9492-9496, 2011.

[40] P. Angelopoulou, S. Baskoutas, A. Jannussis, and R. Mignani, "Caldirola-Kanai Hamiltonian with complex friction coefficient," II Nuovo Cimento, vol. 109, no. 11, pp. 1221-1226, 1994.

[41] R. A. El-Nabulsi, "Fractional calculus of variations from extended Erdelyi-Kober operator," International Journal of Modern Physics A, vol. 23, no. 16, pp. 3349-3361, 2009.

[42] R. A. El-Nabulsi, "Fractional quantum Euler-Cauchy equation in the Schrodinger picture, complexied harmonic oscillators and emergence of complexied Lagrangian and Hamiltonian dynamics," Modern Physics Letters A, vol. 23, no. 28, pp. 3369-3386, 2009.

[43] R. A. El-Nabulsi, "A periodic functional approach to the calculus of variations and the problem of time-dependent damped harmonic oscillators," Applied Mathematics Letters, vol. 24, no. 10, pp. 16471653, 2011.

[44] L. Herrera, L. Núñez, A. Patiño, and H. Rago, “A variational principle and the classical and quantum mechanics of the damped harmonic oscillator," American Journal of Physics, vol. 54, no. 3, pp. 273-277, 1986.

[45] R. Almeida, A. B. Malinowska, and D. F. M. Torres, "Fractional Euler-Lagrange differential equations via Caputo derivatives," in Fractional Dynamics and Control, part 2, pp. 109-118, Springer, New York, NY, USA, 2012.

[46] A. Ya. Helemskii, Lectures and Excercises on Functional Analysis, vol. 233 of Translations of Mathematical Monographs, American Mathematical Society, Providence, RI, USA, 2006.

[47] A. D. Polyanin and A. V. Manzhirov, Handbook of Integral Equations, CRC Press, Boca Raton, Fla, USA, 1998.

[48] M. Klimek, On Solutions of Linear Fractional Differential Equations of a Variational Type, The Publishing Office of Czestochowa University of Technology, Czestochowa, Poland, 2009.

[49] M. Giaquinta and S. Hildebrandt, Calculus of Variations. I, vol. 310 of Grundlehren der Mathematischen Wissenschaften, Springer, Berlin, Germany, 1996.

[50] R. Almeida and D. F. M. Torres, "Calculus of variations with fractional derivatives and fractional integrals," Applied Mathematics Letters, vol. 22, no. 12, pp. 1816-1820, 2009.

[51] G. S. F. Frederico and D. F. M. Torres, "Fractional optimal control in the sense of Caputo and the fractional Noether's theorem," International Mathematical Forum, vol. 3, no. 9-12, pp. 479-493, 2008. 
[52] A. A. Kilbas, H. M. Srivastava, and J. J. Trujillo, Theory and Applications of Fractional Differential Equations, Elsevier, Amsterdam, The Netherlands, 2006.

[53] U. N. Katugampola, "New approach to a generalized fractional integral," Applied Mathematics and Computation, vol. 218, no. 3, pp. 860-865, 2011.

[54] O. P. Agrawal, M. M. Hasan, and X. W. Tangpong, "A numerical scheme for a class of parametric problem of fractional variational calculus," Journal of Computational and Nonlinear Dynamics, vol. 7, no. 2, Article ID 021005, 6 pages, 2012.

[55] R. Almeida, S. Pooseh, and D. F. M. Torres, "Fractional variational problems depending on indefinite integrals," Nonlinear Analysis: Theory, Methods and Applications, vol. 75, no. 3, pp. 1009-1025, 2012.

[56] S. Pooseh, R. Almeida, and D. F. M. Torres, "Expansion formulas in terms of integer-order derivatives for the Hadamard fractional integral and derivative," Numerical Functional Analysis and Optimization, vol. 33, no. 3, pp. 301-319, 2012.

[57] S. Pooseh, R. Almeida, and D. F. M. Torres, "Approximation of fractional integrals by means of derivatives," Computers \& Mathematics with Applications. In press.

[58] L. C. Young, Lectures on the Calculus of Variations and Optimal Control Theory, Foreword by Wendell H. Fleming, W. B. Saunders, Philadelphia, Pa, USA, 1969.

[59] L. Tonelli, "Sur une méthode directe du calcul des variations," Rendiconti del Circolo Matematico di Palermo, vol. 39, no. 1, pp. 233-264, 1915.

[60] L. Cesari, Optimization-Theory and Applications, vol. 17 of Applications of Mathematics (New York), Springer, New York, NY, USA, 1983.

[61] D. F. M. Torres, "Carathéodory equivalence, Noether theorems, and Tonelli full-regularity in the calculus of variations and optimal control," Journal of Mathematical Sciences (New York), vol. 120, no. 1, pp. 1032-1050, 2004. 


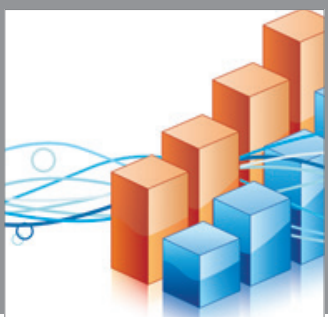

Advances in

Operations Research

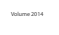

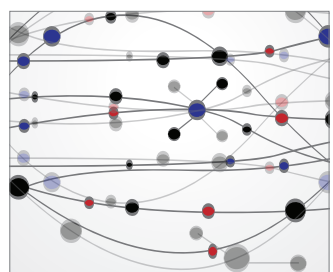

\section{The Scientific} World Journal
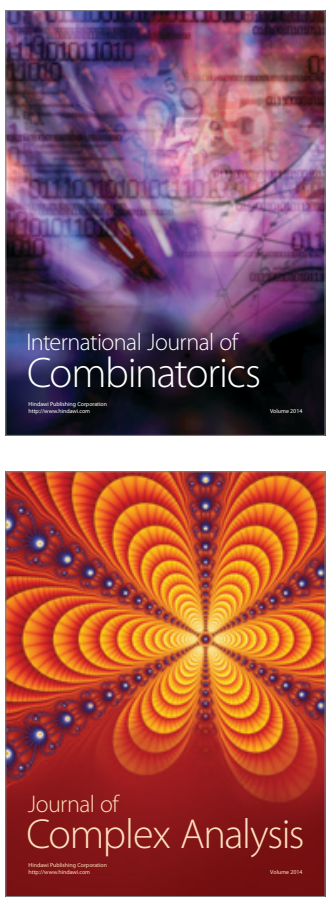

International Journal of

Mathematics and

Mathematical

Sciences
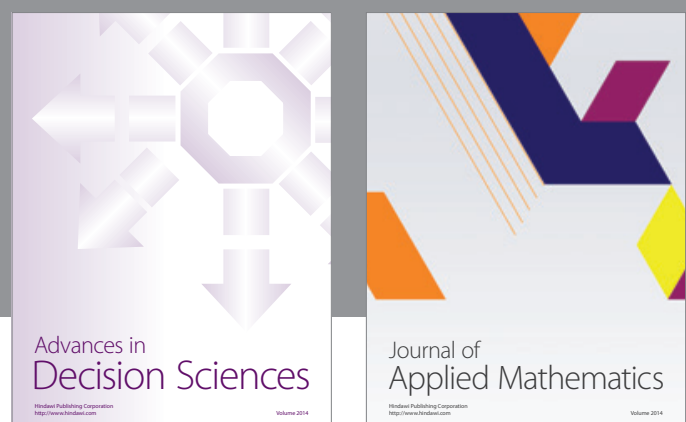

Journal of

Applied Mathematics
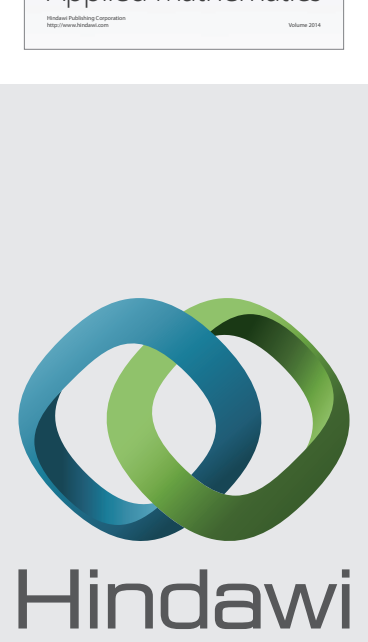

Submit your manuscripts at http://www.hindawi.com
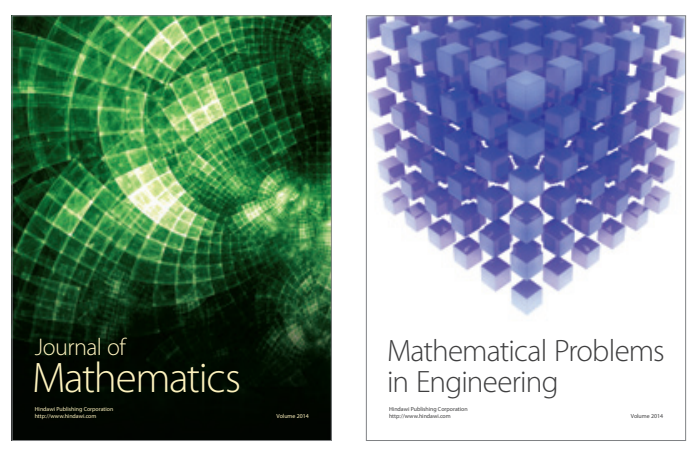

Mathematical Problems in Engineering
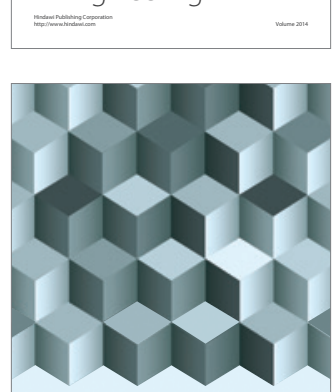

Journal of

Function Spaces
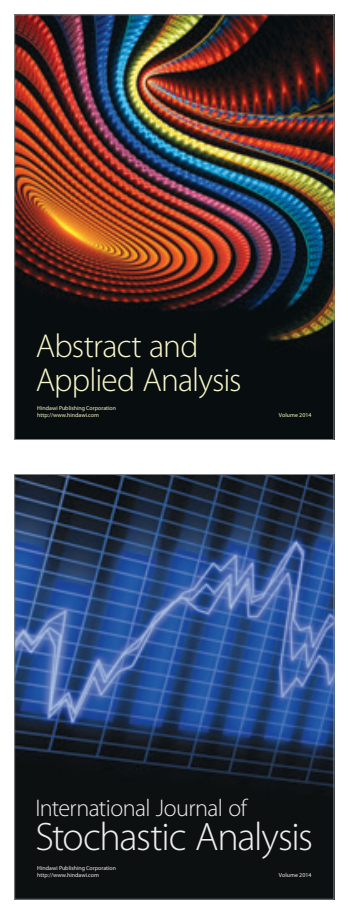

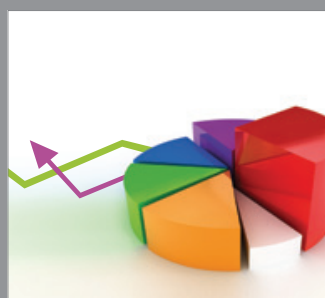

ournal of

Probability and Statistics

Promensencen
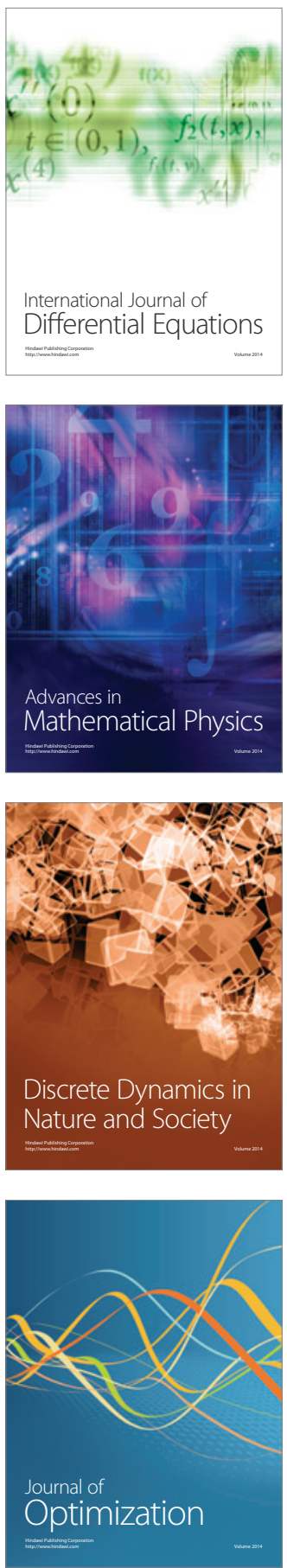